\title{
PREFERENCIAS SOCIALES EN UN JUEGO SENCILLO DE COPARTICIPACION
}

Camilo Rubbini*

Cesar Mateo ***

\section{Resumen}

La evidencia experimental revela que los individuos en sus elecciones se apartan de un comportamiento self-interested. Aquí se presenta un juego experimental sencillo a estudiantes de distintas carreras de la Universidad Nacional de La Plata, que permite contrastar las predicciones de estos modelos canónicos, con representaciones sofisticadas de preferencias sociales. Los resultados encontrados muestran que los agentes incorporan consideraciones de equidad en sus elecciones. En particular, en el escenario considerado, el concepto de equidad parece estar íntimamente ligado al de igualdad.

Clasificación JEL: A 12, A13, C72, C91

Palabras clave: teoría de los juegos - diseño experimental - comportamiento individual - preferencias sociales

\begin{abstract}
Experimental evidence suggests that individuals' choices depart systematically from selfinterest behavior. It reports the results of a simple experimental dictator game played by students from different departments at Universidad Nacional de La Plata to contrast these model predictions with more sophisticated representations of social preferences. The results show that individuals incorporate fairness considerations in their choices. Particularly, in the considered framework, fairness seems to be linked with equality.
\end{abstract}

Jel Classification: A12, A13, C72, C91

Keywords: game theory - experimental design - individual behavior - social preferences

\section{INTRODUCCION}

La vasta literatura sobre Economía experimental revela que los agentes que participan en experimentos, ante diversos tratamientos, realizan elecciones que se apartan de la maximización de sus pagos monetarios, cuando las elecciones afectan los pagos de otros participantes (Kagel y Roth, 1995), (Kahneman, Knetsch y Thaler, 2000). En particular, es

\footnotetext{
* Universidad Nacional de La Plata, Pennsylvania State University. camilorubbini@econo.unlp.edu.ar, camilorubbini@psu.edu

${ }^{* *}$ Universidad Nacional de La Plata
} 
posible observar conductas de sacrificio personal, ya sea para compartir bienestar (dinero) con aquellas personas que se ven desfavorecidas en la asignación previa, como, así también, en casos donde se pretende castigar a quienes han revelado un comportamiento desconsiderado o abiertamente de maltrato. Estos desvíos de las conductas predichas para individuos self-interested ha propiciado el desarrollo de modelos alternativos basados en la consideración de preferencias sociales (Charness y Rabin, 2002). En estos modelos se supone que si bien los individuos están motivados por su interés personal, se preocupan por los efectos de sus elecciones sobre el resto de los agentes.

En este trabajo se analiza en qué medida diferentes modelos propuestos por la literatura de preferencias sociales permiten explicar las respuestas de estudiantes universitarios. Se sigue el enfoque analítico de Charness y Rabin $(1999,2002)$, pero en este caso, e lmarco elegido consiste en un problema hipotético de distribución de fondos entre dos provincias. La elección de tal escenario se justifica en el deseo de avanzar en la comprensión de qué es lo que los individuos consideran justo en el contexto de la coparticipación federal de impuestos. La literatura de la Economía del bienestar ha introducido consideraciones de justicia para evaluar la deseabilidad de diferentes asignaciones, pero comúnmente ha ignorado las preocupaciones de justicia y equidad de los agentes estudiados (Amiel y Cowell, 1999). Es que la Economía del bienestar debe ocuparse no sólo de las asignaciones eficientes de bienes materiales, sino también del diseño de instituciones de manera que los individuos se sientan conformes con la forma en que interactúan unos con otros (Rabin, 1993).conforme a ello, el conocimiento sobre el concepto de justicia de los individuos puede contribuir al estudio de su comportamiento y su bienestar, enriqueciendo finalmente el análisis de política económica y el diseño de instituciones.

En la primera parte del trabajo se sintetizan las representaciones de preferencias consideradas. En segundo lugar, se exponen las características del juego presentado a los estudiantes y del procedimiento empleado. Posteriormente se detallan los resultados obtenidos.

\section{LAS PREFERENCIAS SELF-INTERESTED Y LAS PREFERENCIAS SOCIALES}

Los modelos de preferencias sociales suponen que los individuos están motivados por un interés propio, pero que asimismo demuestran consideración sobre el estado de los demás agentes. En este trabajo se considerarán cuatro representaciones alternativas de preferencias presentadas en Charness y Rabin (2002). Los diferentes tipos de modelos y sus características particulares se pueden sintetizar en:

-Modelos de interés propio: los individuos con estas preferencias sólo se preocupan por optimizar sus propios pagos, independientemente de los pagos del resto de los participantes.

-Modelos de preferencias competitivas: bajo este tipo de preferencias, los individuos muestran interés en alcanzar un resultado (asignación) que los posicione lo mejor posible con relación a los demás, mientras que igualmente se preocupan por los pagos que perciben.

-Modelos de aversión a la diferencia: en estos se asume que los individuos están motivados a reducir las diferencias vigentes entre los pagos propios y ajenos, lo que no implica que entre 
dos asignaciones alternativas se opte necesariamente por aquella en la cual la diferencia entre los pagos sea menor (Fehr y Schmidt, 1999).

-Modelos de bienestar social: se asume que los individuos están interesados en incrementar el excedente social total, aunque prestando una atención especial por aquéllos que presentan bajos pagos. Esta preocupación social implica una diferencia sustancial respecto de una postura benthamista, donde el objetivo consiste en la maximización del excedente social, con la consiguiente omisión de consideraciones distributivas.

De las definiciones precedentes, se desprende que las elecciones individuales guiadas según las preferencias por el bienestar social se dirigirían siempre en el sentido de mejoras paretianas, en búsqueda de alcanzar asignaciones Pareto-eficientes. De este modo, quedarían fuera de su poder explicativo los comportamientos de naturaleza Pareto-destructivos. La consideración de factores de reciprocidad, constituye un complemento natural para la explicación de tales conductas. En efecto, es posible imaginar un aspecto determinante de las motivaciones personales, en la conducta asumida por otros y su incidencia sobre quienes deban pronunciarse respecto de cualquier cuestión ${ }^{1}$.

\section{UN JUEGO SENCILLO DEL DICTADOR: LA COPARTICIPACION FEDERAL DE IMPUESTOS}

El juego sencillo del tipo dictador, es presentado a estudiantes universitarios de las carreras de: Lic. en Economía, Lic. en Administración, Contador Público, Lic. en Ciencias Jurídicas, Lic. en Comunicación Social, Lic. en Sociología, Lic. en Psicología y Trabajo Social. Tales carreras de grado corresponden a la Universidad Nacional de La Plata ${ }^{2}$. La variada formación disciplinaria de los integrantes de la muestra está orientada a analizar si las elecciones tomadas por individuos formados en la teoría de la elección racional con individuos self-interested, se ajustan en mayor medida a las hipótesis formuladas por ésta. Los alumnos que participaron del experimento fueron encuestados durante una clase. A todo alumno se le presento el siguiente problema de elección entre dos alternativas de repartición de fondos entre provincias:

"La ley de coparticipación federal de impuestos determina la distribución primaria de fondos (nación vs. provincias) y la distribución secundaria de fondos (provincia A vs. provincia B). La mejora en las condiciones económicas del país evidenciada en los últimos años, hace posible asignar a cada provincia una cantidad de fondos extra, respecto a lo que cada una recibe actualmente. El gobierno nacional propone dos esquemas alternativos (I y II) de reparto entre dos provincias (A y B) para asignar los nuevos fondos. Usted es ciudadano de la provincia A y debe indicarle a sus legisladores, qué esquema prefiere. En la figura se presentan las sumas de dinero obtenidas por cada provincia bajo cada uno de los esquemas.

\footnotetext{
1 En los modelos de reciprocidad la voluntad por mejorar los pagos ajenos depende de cómo se perciba el comportamiento de los otros. En este marco, el maltrato y la desconsideración pueden alentar en un individuo el deseo de penalizar a quienes así se comporten, incluso, tal vez, a costa del sacrificio propio. El presente experimento sencillo no permite efectuar consideraciones sobre comportamientos recíprocos.

${ }^{2}$ Tanto el protocolo de la encuesta como los datos obtenidos, pueden solicitarse a los autores.
} 


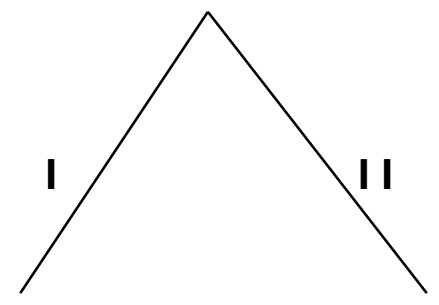
A 400
A 400
B 400
B 750

Así, si elije $I$ la provincia A obtiene 400 y la B obtiene 400. Si, en cambio, elije $I I$, la provincia A obtiene 400 y la B obtiene 750. Elija un esquema".

El juego del tipo dictador presentado consta de ocho versiones, en las que los participantes debieron elegir entre dos alternativas, mutuamente excluyentes. Se realizaron 765 encuestas, obteniéndose una tasa de respuesta del 96,3\% (737 respuestas y 28 abstenciones). La Tabla 1 describe los ocho juegos, la consistencia de cada alternativa con las hipótesis de comportamiento de los diferentes modelos y las respuestas por cada alternativa en cada juego.

Tabla 1 - Consistencia de los juegos con los modelos distributivos

\begin{tabular}{|c|c|c|c|c|c|c|}
\hline & \multirow{2}{*}{ Juego } & \multicolumn{2}{|c|}{ Preferencias } & \multirow{2}{*}{$\begin{array}{c}\text { Encuestas } \\
\text { (737) }\end{array}$} & \multicolumn{2}{|l|}{ Elecciones } \\
\hline & & Izquierda & Derecha & & Izquierda & Derecha \\
\hline 1 & $(400,400)$ vs $(400,750)$ & $\$, \mathrm{C}, \mathrm{DA}$ & $\$, W$ & 92 & 47 & 53 \\
\hline 2 & $(400,400)$ vs $(375,750)$ & $\$, \mathrm{C}, \mathrm{DA}, \mathrm{W}$ & $\mathrm{W}$ & 94 & 75 & 26 \\
\hline 3 & $(200,800)$ vs $(0,0)$ & $\$, \mathrm{C}, \mathrm{DA}, \mathrm{W}$ & C,DA & 91 & 66 & 34 \\
\hline 4 & $(600,300)$ vs $(500,700)$ & $\$, \mathrm{C}, \mathrm{DA}, \mathrm{W}$ & $\mathrm{DA}, \mathrm{W}$ & 88 & 30 & 70 \\
\hline 5 & $(700,200)$ vs $(600,600)$ & $\$, C, D A, W$ & $\mathrm{DA}, \mathrm{W}$ & 92 & 16 & 84 \\
\hline 6 & $(800,0)$ vs $(400,400)$ & $\$, \mathrm{C}, \mathrm{DA}, \mathrm{W}$ & $\mathrm{DA}, \mathrm{W}$ & 92 & 10 & 90 \\
\hline 7 & $(600,300)$ vs $(500,350)$ & $\$, \mathrm{C}, \mathrm{DA}, \mathrm{W}$ & $\mathrm{DA}, \mathrm{W}$ & 90 & 34 & 66 \\
\hline 8 & $(600,300)$ vs $(400,400)$ & $\$, C, D A, W$ & $\mathrm{DA}, \mathrm{W}$ & 98 & 33 & 67 \\
\hline
\end{tabular}

Fuente: Elaboración propia en base a datos de la encuesta realizada a alumnos de la UNLP.

Así, por ejemplo, en el juego 2, la opción izquierda es compatible con los modelos de interés propio (\$), competitivo (C), de aversión por la diferencia (DA) y de bienestar social (W). Por su parte, en el mismo juego, la opción derecha sólo es compatible con el modelo de bienestar social. 


\section{ANÁLISIS DE LOS RESULTADOS}

Cuando se analiza el poder explicativo de las diferentes hipótesis de comportamiento, se encuentra que la representación de las preferencias individuales que mejor ajusta a las elecciones observadas, corresponde a las denominadas como Aversión a la diferencia y de bienestar social, cuyas capacidades para comprender los comportamientos observados resulta significativamente superior a las de las representaciones de las preferencias según los modelos del interés propio y competitivo. Estos resultados se muestran en la siguiente tabla

Tabla 2 -Ajuste del comportamiento según los modelos de preferencias

\begin{tabular}{ccccc}
\hline Encuestas & Interés Propio & Competitivo & $\begin{array}{c}\text { Aversión a la } \\
\text { Diferencia }\end{array}$ & $\begin{array}{c}\text { Bienestar } \\
\text { Social }\end{array}$ \\
\hline 737 & 335 & 317 & 664 & 663 \\
\hline $100 \%$ & $45.5 \%$ & $43.0 \%$ & $90.1 \%$ & $90.0 \%$ \\
\hline
\end{tabular}

Fuente: Elaboración propia en base a datos de la encuesta realizada a alumnos de la UNLP

Tal como se observa, el $90 \%$ de las elecciones realizadas implican un comportamiento que puede ser representado por medio de preferencias que incorporen consideraciones de tipo social, frente a un poder explicativo inferior al 50\% correspondiente a los modelos de preferencias basadas en el interés propio.

Sin embargo, cuando ambas alternativas (de una versión), resultan compatibles con un determinado modelo de preferencias, la elección entre las mismas no dice demasiado respecto del poder explicativo del modelo. Para controlar por este factor, en la Tabla 3 se evalúa la capacidad de las diferentes definiciones de preferencias para explicar el comportamiento cuando cada modelo realiza una predicción compatible únicamente con una sola de las alternativas de la versión del problema.

Tabla 3 - Ajuste del comportamiento según los modelos de preferencias:

Caso en que la predicción de la representación de preferencias es única

\begin{tabular}{c|c|c|c|c|c|c|c}
\hline \multicolumn{2}{c|}{ Interés Propio } & \multicolumn{2}{c|}{ Competitivo } & \multicolumn{2}{c|}{$\begin{array}{c}\text { Aversión a la } \\
\text { Diferencia }\end{array}$} & \multicolumn{2}{c}{$\begin{array}{c}\text { Bienestar } \\
\text { Social }\end{array}$} \\
\hline Elecciones & Posibilidades & Elecciones & Posibilidades & Elecciones & Posibilidades & Elecciones & Posibilidades \\
\hline 243 & 645 & 226 & 646 & 113 & 186 & 109 & 183 \\
\hline \multicolumn{2}{|c|}{$38 \%$} & \multicolumn{2}{|c|}{$35 \%$} & \multicolumn{2}{c|}{$61 \%$} & & $60 \%$ \\
\hline
\end{tabular}

Fuente: Elaboración propia en base a datos de la encuesta realizada a alumnos de la UNLP

En este caso, los resultados son cualitativamente similares a los observados en el de la alternativa anterior, con las representaciones de preferencias que reflejan motivaciones 
sociales demostrando una capacidad explicativa superior a la de los modelos que fundamentan las preferencias en motivaciones individuales. Sin embargo, el grado de ajuste del primer tipo de modelos se reduce al $60 \%$ mientras que para los segundos la pérdida de poder explicativo resulta menor.

Bajo la hipótesis de preferencias basadas en el propio interés, las decisiones de sacrificio individual quedan fuera del alcance explicativo de los modelos de elección. La consideración de motivaciones sociales en la toma de decisiones individuales, como de cuestiones de reciprocidad que afecten el proceso decisorio según el comportamiento de terceros, permiten dar cuenta de tal tipo de conductas. La Tabla 4, muestra la consistencia de las diferentes configuraciones de preferencias con la elección que implica un sacrificio personal. Del total de encuestas realizadas, 645 presentaron una alternativa de esta naturaleza, habiendo la misma sido elegida en 402 oportunidades, lo que representa una tasa de sacrificio del $62,3 \%$.

Tabla 4 - Consistencia de las preferencias con la elección de sacrificio

\begin{tabular}{lccc}
\hline \multicolumn{4}{c}{ Juegos en los que existe posibilidad de sacrificio } \\
\hline & Sacrificios & Posibilidades & $\begin{array}{c}\text { Tasa de } \\
\text { sacrificio }\end{array}$ \\
Total & 402 & 645 & $62.3 \%$ \\
$\begin{array}{l}\text { Cuando el sacrificio es respecto } \\
\text { del modelo: }\end{array}$ & Sacrificios & Posibilidades & Tasa de \\
Competitivo & & & sacrificio \\
Consistente & 31 & 91 & $34.1 \%$ \\
Inconsistente & 371 & 554 & $67.0 \%$ \\
Aversión a la diferencia & & & \\
Consistente & 378 & 551 & $68.6 \%$ \\
Inconsistente & 24 & 94 & $25.5 \%$ \\
Bienestar social & & & \\
Consistente & 371 & 554 & $67.0 \%$ \\
Inconsistente & 31 & 91 & $34.1 \%$ \\
\hline
\end{tabular}

Fuente: Elaboración propia en base a datos de la encuesta realizada a alumnos de la UNLP

Analizando el comportamiento individual de cada representación de preferencias según este tipo de elección, se puede apreciar que en los modelos de Aversión a la diferencia y de Bienestar social, en los casos en que la elección de sacrificio es consistente con la definición del modelo, la aceptación de la misma alcanza tasas del 68,6\% y 67\% respectivamente. Por el contrario, sólo en el $34,1 \%$ de los casos se opta por tal alternativa cuando ésta resulta consistente con el modelo Competitivo (dada la definición que adoptan las 
preferencias en este caso, el individuo se inclinaría por la alternativa que presenta el menor pago propio para inducir una reducción aún mayor en el de la otra parte, de modo de mejorar su posición relativa).

Los resultados anteriores reflejan un fuerte peso de las consideraciones de tipo social, como la reducción de la desigualdad y la optimización del excedente social (siempre que el sacrificio realizado sea eficiente desde una perspectiva paretiana e implique un bajo costo para la parte perjudicada), como fuente de determinación de las preferencias y las consecuentes decisiones de los individuos.

La Tabla 5 permite analizar el impacto sobre la desigualdad de las elecciones de sacrificio, en dos contextos: por un lado, en el marco de juegos que permiten un comportamiento Pareto-destructivo; y por otro, en juegos en los que el sacrificio favorece al prójimo.

Tabla 5 - Sacrificio e impacto sobre la desigualdad

Tipo de Juego

\begin{tabular}{|c|c|c|c|}
\hline-5 & & & Sacrificio \\
\hline Juegos que permiten un comportamiento & 199 & 371 & $53.6 \%$ \\
\hline Pareto- destructivo & & & \\
\hline Reducen la desigualdad & 199 & 371 & $53.6 \%$ \\
\hline Sin efecto & - & - & - \\
\hline Incrementan la desigualdad & - & - & - \\
\hline $\begin{array}{l}\text { Juegos en los que el sacrificio ayuda al } \\
\text { prójimo }\end{array}$ & 371 & 554 & $67.0 \%$ \\
\hline Reducen la desigualdad & 347 & 460 & $75.4 \%$ \\
\hline Sin efecto & - & - & - \\
\hline Incrementan la desigualdad & 24 & 94 & $25.5 \%$ \\
\hline Total de Juegos & 570 & 925 & $61.6 \%$ \\
\hline Reducen la desigualdad & 546 & 831 & $65.7 \%$ \\
\hline Sin efecto & - & - & - \\
\hline Incrementan la desigualdad & 24 & 94 & $25.5 \%$ \\
\hline
\end{tabular}

Fuente: Elaboración propia en base a datos de la encuesta realizada a alumnos de la UNLP.

En el caso de los juegos que presentan la posibilidad de manifestar un comportamiento Pareto-destructivo, se observa una alta tasa de aceptación de tal alternativa, cuando el 
sacrificio propicia una reducción de la desigualdad ${ }^{3}$. La tasa de sacrificio en este caso asciende al 53,6\%. Esta tasa podría considerarse elevada, cuando se piensa que la misma responde a la elección de alternativas socialmente ineficientes, pudiendo ser reflejo de fuertes consideraciones sociales en favor de una mayor igualdad.

Por otra parte, al considerar únicamente los juegos en los que el sacrificio ayuda al prójimo, la tasa de aceptación de la elección de sacrificio, cuando esta favorece una disminución de la desigualdad, es del $75,4 \%$, frente al $25,5 \%$ en los casos en que ésta ocasiona un incremento en la desigualdad. Este último caso se corresponde al de un individuo que elige sacrificarse al estar percibiendo un pago inferior al de un tercero que se ve beneficiado, por lo que detrás de dicha elección subyace un argumento de eficiencia o maximización del excedente social, aún a costa del sacrificio personal y aumento de la desigualdad. Nótese que la tasa de aceptación de la alternativa de sacrificio resulta significativamente inferior de cuando ésta propicia una disminución de la desigualdad, caso que se corresponde con el de un individuo dispuesto a sacrificarse mientras percibe un pago superior al de la otra parte.

\section{DIFERENCIAS EN LAS RESPUESTAS SEGUN EL TIPO DE FORMACION}

Un análisis que es interesante realizar, es el de comparar las respuestas según sea la formación de los encuestados. Para ello, se realizó una prueba $U$ de Mann-Whitney sobre la igualdad en la distribución de las dos poblaciones de encuestados: alumnos de la facultad de Ciencias Económicas (C.E.) y alumnos de diversas facultades de otras Ciencias Sociales (C.S). En las versiones 2, 4, 5 y 8 del problema, el estadístico de la prueba no permitió rechazar la hipótesis de igualdad en las distribuciones poblacionales; mientras que para las versiones 1, 3, 6 y 7, el estadístico de la prueba llevó a rechazar la hipótesis nula de igualdad. En la Tabla 6, se puede observar que los alumnos de C.S. muestran una mayor propensión a elegir las asignaciones más igualitarias, mientras que los alumnos de C.E. tienden a favorecer en mayor medida las asignaciones Pareto-eficientes, y en aquel caso donde las asignaciones implican el mismo nivel de excedente social (versión 6), la elección de estos por la alternativa que optimiza el pago propio es significativamente superior a la proporción de votos que recibe de parte de los estudiantes de C.S.

Tabla 6 -Versiones especiales

\footnotetext{
${ }^{3}$ Quedan por implementar juegos donde la elección de sacrificio genere un incremento de la desigualdad.
} 


\begin{tabular}{ccc}
\hline \multicolumn{3}{c}{ Versión 1: (400,400) vs $(400,750)$} \\
\hline Facultad y Elección & I & II \\
Cs. Económicas & 38.9 & 61.1 \\
Cs. Sociales & 57.9 & 42.1 \\
\hline
\end{tabular}

\begin{tabular}{ccc}
\hline \multicolumn{3}{c}{ Versión 3: $(200,800)$ vs $(0,0)$} \\
\hline Facultad y Elección & I & II \\
Cs. Económicas & 72.2 & 27.8 \\
Cs. Sociales & 56.8 & 43.2 \\
\hline
\end{tabular}

\begin{tabular}{ccc}
\hline \multicolumn{3}{c}{ Versión 6: $(800,0)$ vs $(400,400)$} \\
\hline Facultad y Elección & I & II \\
Cs. Económicas & 15.7 & 84.3 \\
Cs. Sociales & 2.4 & 97.6 \\
\hline
\end{tabular}

\begin{tabular}{ccc}
\hline \multicolumn{3}{c}{ Versión 7: (600,300) vs (400,400) } \\
\hline Facultad y Elección & I & II \\
Cs. Económicas & 46.0 & 54.0 \\
Cs. Sociales & 20.0 & 80.0 \\
\hline
\end{tabular}

Fuente: Elaboración propia en base a datos de la encuesta realizada a alumnos de la UNLP

\section{CONCLUSION}

Los resultados obtenidos son consistentes con una extensa literatura en la cuestión en cuanto a que los individuos se apartan sistemáticamente de elecciones self-interested.

Los modelos de Aversión a las diferencias y de Bienestar social consiguen explicar en mayor medida las elecciones observadas que otras representaciones de preferencias, tanto cuando el modelo hace una única predicción como cuando ambas elecciones son compatibles con el modelo. Estos resultados son consistentes con los presentados en Charness y Rabin (2002) donde, sin embargo, el modelo de Bienestar social tiene un desempeño visiblemente superior al de Aversión a las diferencias. Esto, creemos, se explica en el diferente marco de distribución presentado en su trabajo, donde el intercambio se realiza entre dos individuos en un contexto de mercado con incentivos monetarios. 
En el trabajo se encuentra asimismo una alta tasa de sacrificio personal en las decisiones, lo que se verifica particularmente cuando el sacrificio "ayuda" al prójimo.

Finalmente, en la comparación de las respuestas de alumnos de Ciencias Económicas con los de otras Ciencias Sociales, en los casos en que las respuestas a los problemas son significativamente diferentes, los primeros tienden a favorecer las elecciones que maximizan el excedente social mientras que los segundos tienden a favorecer las alternativas más igualitarias.

\section{BIBLIOGRAFIA}

Amiel, Y. y Cowell, F., A. (1999), “Thinking about Inequality. Personal Judgment and Income Distributions", Cambridge University Press, UK.

Charness, G. Y Rabin, M. (1999), "Social Preferences: Some Simple Tests and a New Model," Universitat Pompeu Fabra and University of California at Berkeley, mimeo. , (2002), "Understanding Social Preferences with Simple Tests." Quarterly Journal of Economics, ago, pp. 817-869.

Fehr, E. y Schmidt K. (1999), "A Theory of Fairness, Competition, and Cooperation." Quarterly Journal of Economics, Vol.114, pp.817-868.

Kagel, J. y Roth, A. (Editors). (1995) The Handbook of Experimental Economics, Princeton University Press, New Jersey.

Kahneman, D. Knetsch, J. y Thaler, R. (2000), Fairness as a Constraint on Profits: Entitlements in the Market, Reprinted in Choices, Values and Frames, Cambridge University Press.

Rabin, M. (1993), "Incorporating Fairness into Game Theory and Economics." American Economic Review, Vol .83, pp. 1281-1302.

, (1998), "Psychology and Economics", Journal of Economic Literature, Vol 36, mar, pp. 11- 46. 